\title{
Network Update
}

\section{Your Own Internet Web Server}

\section{by Tim Neese \& Bill Nyman, University of Colorado, Boulder}

\section{The World-Wide Web Phenomenon}

"Stay with [the Web] long enough, and sooner or later, you are going to want to try your hand at composing."
Three years ago, most of us were busy slogging our way into the Internet, or trying to figure out how much we needed to. Perched on a familiar island of email, we caught glimpses of emerging Internet technologies as they went bobbing on by. Some opined savantly that a new super or "killer" application would appear quite soon, somehow harnessing the full communications potential of the Net and making that potential easy and convenient to exploit. The more wary regarded the Net's decentralized, unstandardized and unplanned growth and development as anarchy-cheerful anarchy perhaps, but anarchy nonetheless. The quieter ones waited to see how all of this was going to do them any good. And a lot of people just weren't paying much attention.

Then came the World-Wide Web. Imagine our surprise to find that everyone had been right all along! The sloggers kept slogging, the savants kept visioning, the wary kept trepidating, the quiet ones still looked on from the sidelines, and the rest. .. are about to stop reading this article.

The Web difference was that we could do it on-line, in hypertext and multimedia. Inspiration and awkwardness flooded the Net, and the browser or Web crawler-a kind of multimedia Internet receiver-crept onto desktops inexorably. Personal Web pages uegan popping up everywhere, complete with awful looking photos of people who should know better, and their pets. Universities and corporations joined the stampede, and the phrase, "You don't have a home page yet?" was a thing to be dreaded. Soon it was not enough for the university to have a Web page; departments and units wanted them, too, along with offices, programs, centers, and individual students and faculty members. 
That was last year. And the Spring of this year. This Fall, no self-respecting, bleeding-edge course would be offered on campus without a Web presence, and in fact, the course Web page is turning out to be a useful and versatile means of organizing materials and promoting communication. The acquisition of Web literacy does seem to follow a pattern. We all start out passively: net surfing and Web browsing, stealing time from more worthwhile pursuits. Some return to their original pursuits because the Web thing is just not for them. Many others immerse themselves in the Web, learning and eventually admiring its varied uses and capabilities. The immersed start to sound like concert-goers at intermission, sometimes casually and sometimes earnestly defending their tastes and how the current fare suits them. Stay with it long enough, and sooner or later, you are going to want to try your hand at composing.

\section{The Basics Perhaps you just want to display a personal Web page, like} an all-seasons Christmas card for family and friends. (You might want to have a trusted friend look over your photos first.) For this, you can probably persuade one of the Large Systems People (or LSP) at your campus computing center to give you some space on one of their UNIX servers that does Web serving. This might be the same computer that now houses your email account. The same strategy will work if you would like to tell the world a little about your lab: who you are, where you are, what you do, and what hours and days you do it. You may want to hire out the authoring of your first page. If your campus is anything like ours, a quick call to the computing center will turn up someone, student or staff, who is well up-to-speed on making Web pages.

\section{Beyond the Basics}

Another way you can begin your career in page authoring is by using a template. This is technical talk for a four step process. Step 1. "Browse a suitable Web page." (Shop around for something good that someone else has done.) Step 2. "View the source code." (Download the page to your computer; this is both doable and legal because you can take a copy of the code for any browsable page without asking, and no one has tackled the copyright thing on the Web yet.) Step 3. "Customize the template to suit your needs." (Change the booty into a treasure of your own.) Step 4. "Cultivate pride of authorship." (Lie about what you've done while bragging to everybody about your creativity.) Here, too, your computing center can probably provide you with someone to assist you. (Get an accomplice.) The computing center will probably have space for your creations on one of their Web servers, too. 


\section{The Big Time}

Prerequisites

Naming Your Server
Serving your Web pages on a remote host is not without its drawbacks. Downloading page code to your desktop constantly or repeatedly gets tedious. Once you've created or edited a page, you may not be able to try it out without uploading it (again). If the page or its links don't turn out right on that pass, you're in for another round of downloading, editing, uploading and auditioning. Sometimes you end up with a file containing the old or original page up on the Web server and an edited or new file on your desktop with the same name in which case you are only an interruption away from confusion. And finally, the computing center starts to get peeved because you have too much material or your web site is generating so much interest that the traffic on their network is getting too heavy. The more changes you make, the more frequently you make them, and the larger your set of Web pages grows, the more likely you are to wish for direct access to page code and local control of the whole process. These are some of the advantages of having your own Internet Web server.

Most campuses in 1995 support TCP/IP, the Internet protocol. (If yours doesn't, it's time to scream-loudly!) Most labs contain one of two kinds of local area network servers: Novell or Appletalk. The odds are very high that your network is Ethernet-based and connected to a building network. That building network in turn empties into the central "backbone" for your institution, and if you've made it this far, you probably have a campus gateway to the Internet, too. If all this so, you're in business. (If you're not sure about your network, contact a Large Systems Person at your computing center and tell them about your Web plans. If they reply that all the network technology is there, but they never heard of a Novell or an Appletalk Web server, thank them politely and then read on.) That Novell or Appletalk server of yours- the one that has stored and shared your files and queued your printers-is about to learn a new trick. It will be your own Internet Web server.

Every computer on a TCP/IP based network has a numeric address, such as 128.138.231.172. For Web browsing, most people prefer something more meaningful and much easier to remember. Domain Name Service (or DNS) provides a way for your Large Systems People to assign your network server 
a Fully Qualified Hostname (or FQH) in addition to its numeric Internet Protocol (IP) address. This name can tell the world something about who you are and what you are up to, and maybe stick in peoples' minds long enough for them to make a bookmark (a listing of your server's address in their browser software). One of the Novell servers we used as a model for this article is located in Academic Media Services at the University of Colorado. Its name is ams.colorado.edu. Similarly, our test-case Appletalk server is named masala.colorado.edu. Think about giving your server a name like this before its Web debut. Keep in mind that the name you choose must be unique, and must conform to certain conventions and standards. Your local LSP can tell you all about these.

Novell Server Software
There are two products for you to consider when adding Web server capabilities to your Novell network server: HTTPD from GLACI (Great Lakes Area Commercial Internet) and the new Netware Web Server from Novell.

HTTPD Version 2.0, which appeared in mid-November, is the most recent in a series of dependable, capable, low-cost Web service software packages written by the GLACI gentlefolk. HTTPD 2.0 handles HTML documents with inline graphics, sound and animation, and supports Multipurpose Internet Mail Extensions (MIME). These are enhancements to the popular Simple Mail Transfer Protocol (SMTP) for text and permit additional types of data to be shared over the Internet, such as binary, audio, and video files. Also included is ISMAP clickable images support so that one graphic image on a Web page can be divvied up into multiple clickable parts. You can set up special lists that allow or deny access to certain users or groups of users based on their IP addresses, a handy security feature.

Another is the Personal Web Pages option. People on your local area network can store their own personal Web page in their password-protected home directory on the server and grant Web access to that page-and only to the page- to the rest of the world. What about scripting and forms? GLACI distributes a PERL module free with each copy of Version 2.0, although it is written by another company. It performs fine for us, and we have heard no complaints about it. Common Gateway Interface (CGI) scripting support was added to this version by GLACI. Because CGI was developed for UNIX servers and then transplanted to Novell, the GLACI CGI has a few quirks, but they are manageable. 
"There are two products for you to consider when adding Web server capabilities to your Novell network server: HTTPD ... and the new Netware Web Server from Novell."
The cost of a single server educational license is a nominal $\$ 236$, and upgrades are free. Version 2.0 assumes that your Novell server is a 386, 486 or Pentium machine running Netware 3.11, 3.12, 4.01 or 4.1 with the Novell TCP/IP Network Loadable Module (NLM) installed. We have tried it on 486 and Pentium machines under 3.12 and 4.1., and have heard about it running fine on others. The consensus opinion is that GLACI-HTTPD is a good citizen, and does not foul up servers or interfere with other NLM's. GLACI welcomes you to try HTTPD 2.0 for free. Point your browser at http:/ / www.glaci.com/info/glaci-httpd.html and download a 30day license to a floppy disk on your workstation. Plug the floppy into your server, run the install utility, and you'll be Web serving in minutes. The software occupies less than $500 \mathrm{~K}$ of disk space and is thrifty with your RAM. You can get free technical support by phoning 415-475-6388 (that's in Wauwatosa, Wisconsin), or by sending email to support@glaci.com. You can see GLACI-HTTPD in action on http:/ /ams.colorado.edu - one of our model servers.

Netware Web Server is a brand new product from Novell, so what we have to report at this writing are the official rumors. Novell tells us that, like GLACI-HTTPD, Web Server handles HTML documents with in-line graphics, sound and animation. Early press releases do not mention MIME and ISMAP support by name, but we suspect they are probably built-in. Unlike GLACI-HTTPD, Web Server requires Netware 4.1, a potential disadvantage for Netware 3.xx devotees, but also leverages the Netware Directory Services (NDS) capabilities of Netware 4.1 into its Web serving role to enhance security and flexibility of access. Like GLACI-HTTPD, Web Server allows you to establish IP address based security checklists, and includes a Common Gateway Interface module called R-CGI, as well as a PERL Script Interpreter. Whether or not the latter two work just like their UNIX counterparts is unclear. This package includes a BASIC Script Interpreter as well. Other details include: 10-minute installation and configuration, on-line manuals and help, a copy of Netscape Navigator (which is freeware anyway), and nepotistically close integration with WordPerfect's Internet Publisher (although the small print says other HTML publishers will work fine, too). Web Server will be for sale through Authorized Novell Channel Partners on December 21, 1995 and lists for a $\$ 995$. If pricing follows the usual Novell policy, educational users should be able to get a license for about $\$ 800$. No clue on what upgrades may run, but it's fair to say that Novell is not known for its largesse. Netware 4.1 is required for run- 


\section{Macintosh Software}

"While most of the Web servers on the Internet are UNIX machines, about $10 \%$ are running on Macintoshes using MacHTTP or WebStar." ning Web Server, along with 2+ megabytes of disk space to hold the software itself and the on-line documentation. For more late-breaking news, point your browser at http:// corp.novell.com/announce/webserve.

MacHTTP has long been the Web server of choice for Macintosh computers. While most of the Web servers on the Internet are UNIX machines, about $10 \%$ are running on Macintoshes using MacHTTP or WebStar. Ease of setup, maintenance, and editing of Web documents are the primary advantages that lead some to use a Macintosh for a Web server. Low hardware costs for an entry-level Power Macintosh and the ability to use existing equipment, such as an AppleShare file server, likewise lead people to foray into Web publishing on a Macintosh server. Product information and evaluation copies of MacHTTP can be obtained at http:// www.starnine.com/machttp/machttp.html. MacHTTP costs $\$ 65$ for educational institutions, and provides adequate features and performance for people wanting to make just text and graphics available on the Web. It provides security features such as restricting access to particular ranges of IP addresses and assigning server passwords. While it does support AppleScript, CGI, Imagemaps, and MacPerl to offer a variety of interactive forms capabilities through scripting, it can become sluggish when several people access these scripts concurrently. It does perform adequately, however, for sites receiving hundreds of requests or "hits" an hour, which means only about a dozen requests concurrently. Although a Power Macintosh is recommended, our model Macintosh servermasala.colorado.edu - is just an old SE/30 that receives about 500 requests a day and rarely makes more than six concurrent connections. MacHTTP takes less than 1.5 megabytes of RAM and less than 1.0 megabytes of disk space, so it will run comfortably on System 7 and 8 megabytes of RAM.

WebStar is the commercial incarnation of MacHTTP from StarNine Technologies, available on the Web at http:// www.starnine.com/webstar/ and offers performance enhancements and additional features. StarNine claims that WebStar is three to four times faster than MacHTTP 2.0 and competes favorably with many UNIX servers. A Secure Sockets Layer (SSL) extension is available for WebStar to enable a Macintosh server to provide encrypted access for secure transactions such as for electronic billing functions. It also supports 
the full range of scripting and clickable map features to create more interactive and dynamic Web sites. WebStar is best suited for busier sites that receive thousands of connections per hour. Educational pricing starts at $\$ 295$ per copy.

FTPd. Recently, Peter N. Lewis, of Macintosh shareware fame, added Web serving capability to his popular FTP and Gopher server software, FTPd. FTPd allows your Macintosh to make files available via File Transfer Protocol (FTP), text documents accessible via Gopher, and Web pages browsable via the World Wide Web - all for just $\$ 10$ at http:// hyperarchive.lcs.mit.edu/HyperArchive/Archive/comm/ tcp/ftpd-300.hqx. Since it uses the Users and Groups privileges from Apple File Sharing, it requires a Macintosh running System 7 and works well on AppleShare servers. It doesn't support CGI, forms scripting, or clickable maps, but it is adequate for making just text and graphics available on the Web.

Conclusion Novell and Macintosh servers offer an inexpensive way to publicize your services and resources, and make course materials available widely across your campus or to the world. Using your local area network file server as a Web server allows you to edit Web documents in the same way you edit word-processing documents, and spares you the task of constantly transferring files to and from a UNIX server. You can probably use existing equipment, and the software can be set up in minutes and maintained even by ordinary mortals. As long as you make sure that your file and folder names conform to UNIX naming conventions, files stored on a Novell or Macintosh Web server can easily be moved to a UNIX server later if you outgrow your own Internet Web server.

Tim Neese is the Director of the Instructional Technology Resource Center at the University of Colorado at Boulder. His Web page is available at http://spot.colorado.edu/ neese/Home.html.

Bill Wyman, who is really a German teacher, heads the Computing Support Division of Academic Media Services at the University of Colorado at Boulder. 


\section{Computer Assisted Language Learning}

\section{An International Journal}

\section{Editor:}

Keith Cameron, School of Modern Languages, The University, Exeter, England

\section{Audience:}

Teachers and researchers, linguists, computer scientists, psychologists, and educationalists.

\section{Aims and Scope:}

Computer Assisted Language Learning is an international journal which leads the field in its total dedication to all matters associated with the use of computers in language learning (L1 and L2). It provides a forum to discuss the discoveries in the field and to exchange experience and information about existing techniques. The scope of the Journal, by its very definition, embraces a multitude of disciplines.

Areas covered:

$$
\text { - Pedagogical principles and their applications }
$$

- Second Language Research

- Cognitive Modelling

- Observations on, and evaluation of, CALL software

- Intelligent Tutoring Systems

- Digitised versions of Video and Audio

- Application of AI to language teaching

- Computer assisted translation

- Computer assisted composition

- Multi-lingual systems

- Simulated-active learning environments

ISSN 0958-8221

1995 , volume 8,4 issues

Price

Institutions Dfl. 286,00

Individuals Dfl. 126,00

Volumes 1-7 are available from the publisher

Please send/fax your order, or free sample copy request to:

Swets \& Zeitlinger

P.O. Box 825

2160 SZ Lisse

the Netherlands

Tel. (+31) 2521-35111, Fax (+31) 2521-15888 